\title{
Nonlinear Behaviors of Bandpass Sigma Delta Modulators with Stable System Matrices
}

\author{
Charlotte Yuk-Fan Ho ${ }^{1}$, Student Member, IEEE, Bingo Wing-Kuen Ling ${ }^{2}$, Joshua D. Reiss ${ }^{1}$, and \\ Xinghuo $\mathrm{Yu}^{3}$, Senior Member, IEEE
}

\begin{abstract}
It has been established that a class of bandpass sigma delta modulators (SDMs) may exhibit state space dynamics which are represented by elliptical or fractal patterns confined within trapezoidal regions when the system matrices are marginally stable. In this paper, it is found that fractal or irregular chaotic patterns may also be exhibited in the phase plane when the system matrices are strictly stable.
\end{abstract}

Index Terms-Fractals, chaos, bandpass sigma delta modulators, stable system matrix.

\section{INTRODUCTION}

$\mathrm{B}$ ANDPASS sigma delta modulators (SDMs) have many industrial and engineering applications because many systems are required to perform analog to digital conversions on bandpass signals [1]. By using bandpass SDMs, simple and relatively low precision analog components could achieve the objectives. Because of this advantage, this area draws much attention from the researchers in the community. Consequently, some methods for the analysis [6], [7] and design of bandpass SDMs have been proposed [2]-[5].

Since the quantizer in the feedback loop of bandpass SDMs introduces nonlinearities, limit cycles [6] and chaos [7] may occur. Some researchers utilize the nonlinear behavior to suppress unwanted tones from the quantizers [9]-[11]. The most common existing method is to place some unstable poles in the system matrices, so that chaotic behaviors will be exhibited in the systems, and the rich frequency spectra of these chaotic output signals break down the dominant oscillations at the outputs. However, by placing some unstable poles in the system matrices, the stability of the systems is degraded.

In the practical situation, there are leakages on the integrators [8]. This originates from the internal resistances of the components. Even though the leakages may sometimes be

Manuscript received January 24, 2005; revised August 17, 2005. This work was supported by a research grant from Queen Mary, University of London.

Bingo Wing-Kuen Ling is with the department of Electronic Engineering, King's College London (e-mail: wing-kuen.ling@kcl.ac.uk).

Charlotte Yuk-Fan Ho and Joshua D. Reiss are with the department of Electronic Engineering, Queen Mary, University of London.

Xinghuo is with the School of Electrical and Computer Engineering, Royal Melbourne Institute of Technology.

Copyright (c) 2006 IEEE. Personal use of this material is permitted. However, permission to use this material for any other purposes must be obtained from the IEEE by sending an email to pubs-permission@ieee.org. negligible, engineers and circuit designers may impose leakage on the integrators so as to improve the stability of the overall systems. Therefore, the eigenvalues of the system matrices are strictly inside the unit circle, and the system matrices are actually strictly stable.

Although there are some analytical results on the bandpass SDMs [7], most analysis is based on marginally stable system matrices only. For the bandpass SDMs with strictly stable system matrices, the existing results are primarily concerned with limit cycles, but not with fractal or irregular chaotic behavior. In this paper, we show that fractal or irregular chaotic behavior may also occur.

The organization of the paper is as follows. The analytical and simulation results of bandpass SDMs with strictly stable system matrices are given in Section II. Discussion and conclusion are given in Section III.

\section{ANALYTICAL AND SimUlation RESUltS}

The bandpass SDMs in [12] with leakages can be modeled as follows:

$$
\mathbf{x}(k+1)=\mathbf{A x}(k)+\mathbf{B}(\mathbf{u}(k)-\mathbf{s}(k)) \text { for } k \geq 0,
$$

where $\mathbf{x}(k) \equiv\left[\begin{array}{ll}x_{1}(k) & x_{2}(k)\end{array}\right]^{T}$ is the state vector of the system, $\mathbf{u}(k) \equiv\left[\begin{array}{ll}u(k-2) & u(k-1)\end{array}\right]^{T}$ is a vector containing the past two consecutive points from the input signal $u(k)$,

$$
\mathbf{A} \equiv\left[\begin{array}{cc}
0 & 1 \\
-r^{2} & 2 r \cos \theta
\end{array}\right]
$$

is the system matrix of the system,

$$
\mathbf{B} \equiv\left[\begin{array}{cc}
0 & 0 \\
-r^{2} & 2 r \cos \theta
\end{array}\right]
$$

is the matrix associated with the nonlinearity, and

$$
\mathbf{s}(k) \equiv\left[\begin{array}{ll}
Q\left(x_{1}(k)\right) & Q\left(x_{2}(k)\right)
\end{array}\right]^{T} \text { for } k \geq 0,
$$

in which the superscript ${ }^{T}$ denotes the transpose operator,

$$
Q(y) \equiv\left\{\begin{array}{cc}
1 & y \geq 0 \\
-1 & \text { otherwise }
\end{array},\right.
$$

$\theta \notin\{-\pi, 0, \pi\}$ and $0<r<1$. As opposed to standard lowpass SDM systems, bandpass SDMs are designed to operate on high-frequency narrowband signals by shaping the noise from some frequency $f_{0}$ [7], where $f_{0}=\frac{\theta f_{s}}{2 \pi}$, in which $f_{s}$ denotes the sampling frequency. At the desired frequency $f_{0}$, it has noise transfer function zero and signal transfer function 1 [7]. When $\theta \in\{-\pi, 0, \pi\}$, the system is either a lowpass SDM or a 
highpass SDM, which is out of the scope of the paper. The leakage of the system depends on the values of $r$. If $r$ is closer to 0 , then the poles are closer to the origin and the leakage is more serious. If $r$ is closer to 1 , then the poles are closer to the unit circle and the leakage is less significant. For an ideal lossless bandpass SDMs, $r=1$, the system reduces to that described in [12], and the system matrices are marginally stable. Since

$$
\mathbf{s}(k) \in\left\{\left[\begin{array}{ll}
1 & 1
\end{array}\right]^{T},\left[\begin{array}{ll}
1 & -1
\end{array}\right]^{T},\left[\begin{array}{ll}
-1 & 1
\end{array}\right]^{T},\left[\begin{array}{ll}
-1 & -1
\end{array}\right]^{T}\right\} \text { for } k \geq 0,
$$

the value of $\mathbf{s}(k)$ can be viewed as symbols, and $\mathbf{s}(k)$ is called a symbolic sequence.

In this paper, we only consider the cases when $\mathbf{x}(k)$ and $u(k)$ are real signals, that is $\mathbf{x}(k) \in \mathfrak{R}^{2}$ and $u(k) \in \mathfrak{R}$. We also assume that $u(k)$ is a constant input, that is $\mathbf{u}(k)=\mathbf{u}$ for $k \geq 0$.

\section{A. Limit cycle behaviors}

Define

$$
\mathbf{D} \equiv\left[\begin{array}{cc}
r e^{j \theta} & 0 \\
0 & r e^{-j \theta}
\end{array}\right]
$$

and

$$
\mathbf{T} \equiv\left[\begin{array}{cc}
\frac{1}{\sqrt{r}} e^{-\left(\frac{j \theta}{2}\right)} & \frac{1}{\sqrt{r}} e^{\frac{j \theta}{2}} \\
\sqrt{r} e^{\frac{j \theta}{2}} & \sqrt{r} e^{-\left(\frac{j \theta}{2}\right)}
\end{array}\right] .
$$

Since $\theta \notin\{-\pi, 0, \pi\}, \mathbf{T}^{-1}$ exists. As $\mathbf{A}$ is a full rank matrix because $r \neq 0, \quad \mathbf{A}$ can be decomposed via eigen decomposition. That is:

$$
\mathbf{A}=\mathbf{T D T}^{-1} \text {. }
$$

Let $M$ be the period of the steady state of the output sequences (if it exists), that is

$$
\mathbf{s}\left(k_{0}+M+i\right)=\mathbf{s}\left(k_{0}+i\right) \forall i \geq 0,
$$

in which $M \in Z^{+}$and $k_{0} \in Z^{+} \cup\{0\}$. Define

$$
\mathbf{x}_{0}^{*} \equiv \sum_{n=0}^{M-1} \mathbf{T D}^{M-1-n}\left(\lim _{p \rightarrow+\infty} \sum_{m=0}^{p-1} \mathbf{D}^{m M}\right) \mathbf{T}^{-1} \mathbf{B}\left(\mathbf{u}-\mathbf{s}\left(k_{0}+n\right)\right)
$$

and

$$
\mathbf{x}_{i}^{*} \equiv \mathbf{A}^{i} \mathbf{x}_{0}^{*}+\sum_{m=0}^{i-1} \mathbf{A}^{i-1-m} \mathbf{B}\left(\mathbf{u}-\mathbf{s}\left(k_{0}+m\right)\right) \text { for } i=1,2, \cdots, M-1 .
$$

We have the following lemma.

Lemma 1

The following statements are equivalent:

i) $\mathbf{s}\left(k_{0}+M+i\right)=\mathbf{s}\left(k_{0}+i\right) \forall i \geq 0$.

ii) $\lim _{k \rightarrow+\infty} \mathbf{x}\left(k M+k_{0}+i\right)=\mathbf{x}_{i}^{*}$ for $i=0,1, \cdots, M-1$.

iii) $\mathbf{x}(0) \in \Xi_{1} \equiv\left\{\mathbf{x}(0): \exists k_{0} \in Z^{+} \bigcup\{0\}\right.$ such that $\forall k \geq 0$, and

$\left.i=0,1, \cdots, M-1, Q\left(\mathbf{x}\left(k M+k_{0}+i\right)\right)=Q\left(\mathbf{x}_{i}^{*}\right)\right\}$.

Proof:

For i) implies ii), from equation (1), we have:

$\forall p, M \in \mathrm{Z}^{+}$and $\forall k \geq 0$,

$$
\mathbf{x}(k+p M)=\mathbf{A}^{p M} \mathbf{x}(k)+\sum_{n=0}^{p M-1} \mathbf{A}^{p M-1-n} \mathbf{B}(\mathbf{u}-\mathbf{s}(k+n)) .
$$

From equation (9) and (i), we have:

$$
\begin{aligned}
& \mathbf{x}\left(k_{0}+p M\right)=\mathbf{T D}^{p M} \mathbf{T}^{-1} \mathbf{x}\left(k_{0}\right)+ \\
& \sum_{n=0}^{M-1} \mathbf{T} \mathbf{D}^{M-1-n}\left(\sum_{m=0}^{p-1} \mathbf{D}^{m M}\right) \mathbf{T}^{-1} \mathbf{B}\left(\mathbf{u}-\mathbf{s}\left(k_{0}+n\right)\right)
\end{aligned}
$$

Hence, we have:

$$
\lim _{p \rightarrow+\infty} \mathbf{x}\left(k_{0}+p M\right)=\mathbf{x}_{0}^{*} .
$$

By substituting equation (15) into equation (1), the result follows directly.

For ii) implies i), since

$$
\lim _{k \rightarrow+\infty} \mathbf{x}\left(k M+k_{0}+i\right)=\mathbf{x}_{i}^{*} \text { for } i=0,1, \cdots, M-1,
$$

then $\exists k_{1} \geq 0$ such that

$$
Q\left(\mathbf{x}\left(k M+k_{0}+i\right)\right)=Q\left(\mathbf{x}_{i}^{*}\right) \text { for } k \geq k_{1} \text { and } i=0,1, \cdots, M-1 .
$$

Hence, the result follows directly.

For ii) implies iii), since

$$
\lim _{k \rightarrow+\infty} \mathbf{x}\left(k M+k_{0}+i\right)=\mathbf{x}_{i}^{*} \text { for } i=0,1, \cdots, M-1,
$$

then $\exists k_{1} \geq 0$ such that

$$
Q\left(\mathbf{x}\left(k M+k_{0}+i\right)\right)=Q\left(\mathbf{x}_{i}^{*}\right) \text { for } k \geq 0 \text { and for } i=0,1, \cdots, M-1 \text {. (19) }
$$

Hence, the result follows directly.

For iii) implies i), since

$Q\left(\mathbf{x}\left(k M+k_{0}+i\right)\right)=Q\left(\mathbf{x}_{i}^{*}\right)$ for $k \geq 0$ and for $i=0,1, \cdots, M-1,(20)$

the result follows directly.

This completes the whole proof of the lemma.

Lemma 1 associates the steady state of periodic output with a specific set of initial conditions and a corresponding dynamical behavior of the system. According to Lemma 1, we can easily see that the trajectories will converge to the set of fixed points $\left\{\mathbf{x}_{0}^{*}, \mathbf{x}_{1}^{*}, \cdots, \mathbf{x}_{M-1}^{*}\right\}$, and the periodicity of the steady states of the output sequence is equal to the number of fixed points on the phase plane. That implies that all the fixed points (more than or equal to 2) cannot be in the same quadrant. For example, if $M=2$, then there are two fixed points on the phase plane and these two fixed points are located in different quadrants.

The significance of Lemma 1 is that it provides useful information for estimating the periodicity of the steady state of output sequences via the phase portrait. Moreover, Lemma 1 provides useful information to the SDM designers to avoid limit cycle behavior.

It is worth noting that although the state vector is converging to a periodic orbit, it never reaches these periodic points. That means, the state vector is aperiodic even though the output sequence is eventually periodic. This result is different from the case when $r=1$ and $\theta$ is a rational multiple of $\pi$.

Moreover, although $\mathbf{x}_{i}^{*}$, for $i=1,2, \cdots, M-1$, depends on $\mathbf{s}(i)$, for $i=1,2, \cdots, M-1$, it does not depend on $\mathbf{x}(0)$ directly. That is, the fixed points leading to a given symbol sequence are not directly depended on the initial conditions.

When $M=1$, the output sequence will become constant and there is only one single fixed point on the phase portrait. The trajectory will converge to this fixed point, denoted as $\mathbf{x}^{*}$. The significance of this result is that it allows SDM designers to 
avoid fixed point behavior.

It is worth noting that the state vectors of the corresponding linear system will converge to $(\mathbf{I}-\mathbf{A})^{-1} \mathbf{B u}$, which is not the same as that of $\mathbf{x}^{*}$. Comparing these two values, there are DC shifts and the DC shifts are exactly dropped at the output sequences, that is:

$$
(\mathbf{I}-\mathbf{A})^{-1} \mathbf{B u}-\mathbf{x}^{*}=(\mathbf{I}-\mathbf{A})^{-1} \mathbf{B s}_{k_{0}},
$$

in which

$$
\mathbf{s}(k)=\mathbf{s}_{k_{0}} \text { for } k \geq k_{0} .
$$

In addition, this phenomenon is quite different from the case of lowpass SDMs. In such a situation, the average output sequence will approximate the input values even though limit cycle behavior occurs.

Although the nonlinearity is always activated, the rate of convergence only depends on $r$ when the output sequence becomes steady. This is because the DC terms do not affect the rate of convergence. However, if we look at the transient response of the system, that is, the time duration when the output sequence is not constant, the system dynamics could be very complex.

Figure 1 shows the response of the state variables of a bandpass SDM with

$r=0.9999, \theta=\cos ^{-1}(-0.158532), \mathbf{u}=-0.3\left[\begin{array}{ll}1 & 1\end{array}\right]^{T}$ and $\mathbf{x}(0)=\left[\begin{array}{ll}0 & 0.5\end{array}\right]^{T} \cdot(23)$

The state variables will converge to the same fixed value and the output sequences will become constant for $k \geq 2154$.

Figure 2 shows the state trajectory of a bandpass SDM with $r=0.99, \theta=\cos ^{-1}(-0.158532), \mathbf{u}=-0.3\left[\begin{array}{ll}1 & 1\end{array}\right]^{T}$ and $\mathbf{x}(0)=\left[\begin{array}{ll}0 & 0.5\end{array}\right]^{T} \cdot(24)$ The state trajectory will converge to two fixed points and the output sequences are periodic with period 2 for $k \geq 3$.

Although Lemma 1 gives the necessary and sufficient conditions for the occurrence of limit cycles, it is not easy to check whether a periodic sequence is admissible or not. To address this issue, define

$$
\mathbf{s} \equiv\left[\begin{array}{llll}
\mathbf{s}\left(k_{0}\right)^{T} & \mathbf{s}\left(k_{0}+1\right)^{T} & \cdots & \mathbf{s}\left(k_{0}+M-1\right)^{T}
\end{array}\right]^{T},
$$

and

$$
\mathbf{K} \equiv\left[\begin{array}{cccc}
\mathbf{A}^{M-1} & \cdots & \mathbf{A} & \mathbf{I} \\
\mathbf{I} & \ddots & & \mathbf{A} \\
\vdots & \ddots & \ddots & \vdots \\
\mathbf{A}^{M-2} & \cdots & \mathbf{I} & \mathbf{A}^{M-1}
\end{array}\right]
$$

Lemma 2

If the periodic sequence is admissible, then

$$
Q\left(\begin{array}{lll}
{\left[\left((\mathbf{I}-\mathbf{A})^{-1} \mathbf{B u}\right)^{T}\right.} & \ldots & \left.\left((\mathbf{I}-\mathbf{A})^{-1} \mathbf{B u}\right)^{T}\right]^{T} \\
-\mathbf{K}\left(\operatorname{diag}\left\{\left(\mathbf{I}-\mathbf{A}^{M}\right)^{-1} \mathbf{B}, \cdots\left(\mathbf{I}-\mathbf{A}^{M}\right)^{-1} \mathbf{B}\right\}\right) \mathbf{s}
\end{array}\right)=\mathbf{s} .
$$

Proof:

From Lemma 1, if the periodic sequence is admissible, then the state vectors will converge to

$\mathbf{x}_{i}^{*}=(\mathbf{I}-\mathbf{A})^{-1} \mathbf{B u}-\sum_{j=0}^{M-1} \mathbf{A}^{\bmod (i-1-j, M)}\left(\mathbf{I}-\mathbf{A}^{M}\right)^{-1} \mathbf{B s}\left(k_{0}+j\right)$, for $i=0,1, \cdots, M-1$.

Hence, equation (27) is satisfied and this completes the proof.

The importance of Lemma 2 is that it provides information to check whether a periodic sequence is admissible or not, and hence it can conclude whether limit cycles occur or not from the filter parameters.

\section{B. Fractal or irregular chaotic behaviors}

Equation (27) can be expressed as follows:

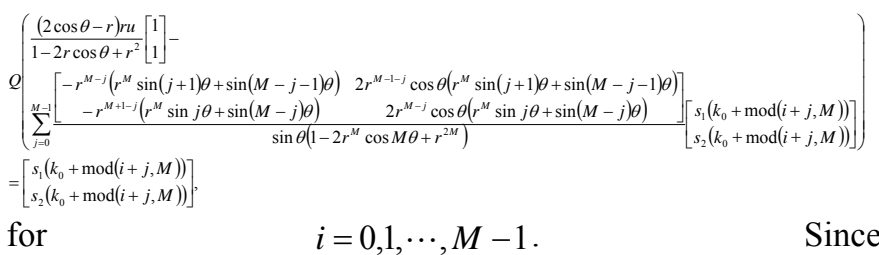

$\mathbf{s}(k) \in\left\{\left[\begin{array}{ll}1 & 1\end{array}\right]^{T},\left[\begin{array}{ll}1 & -1\end{array}\right]^{T},\left[\begin{array}{ll}-1 & 1\end{array}\right]^{T},\left[\begin{array}{ll}-1 & -1\end{array}\right]^{T}\right\}$ for $k \geq 0$, it can be checked that Lemma 2 is not universal satisfied for all filter parameters. That means, there exists some values of $r \in(0,1)$ and $\theta \notin\{-\pi, 0, \pi\}$ such that equation (29) is not satisfied. In this case, the output sequence is aperiodic and the SDM exhibits neither convergent nor limit cycle behaviors at the steady state. Hence,

$$
\Xi_{2} \equiv \mathfrak{R}^{2} \backslash \Xi_{1} \neq \varnothing
$$

where $\varnothing$ denotes the empty set. That means, there exists some initial conditions that the SDM may exhibit either elliptical fractal or irregular chaotic patterns.

Figures 3a-3c show the state trajectories of a bandpass SDM with filter parameters $r=1-10^{-6}$ and $\theta=\cos ^{-1}(-0.158532)$, input step size $\mathbf{u}=-0.3\left[\begin{array}{ll}1 & 1\end{array}\right]^{T}$ and initial conditions $\mathbf{x}(0)=\left[\begin{array}{ll}0 & 0.5\end{array}\right]^{T}, \quad \mathbf{x}(0)=\left[\begin{array}{ll}0 & 0\end{array}\right]^{T}$ and $\mathbf{x}(0)=\left[\begin{array}{ll}1 & 0\end{array}\right]^{T}$, respectively. It can be seen from the figures that fractal patterns are exhibited on the phase plane and the trajectories neither converge to the boundaries of the trapezoids nor any fixed points in the phase portrait. Measurements of the fractal dimension are estimated at 1.78 for the box counting dimension, 1.75 for the information dimension, and 1.72 for the correlation dimension for all these three initial conditions. Figures $3 \mathrm{~d}-3 \mathrm{f}$ show the state trajectories of a bandpass SDM with filter parameters $r=0.9999$ and $\theta=0.01$, input step size $\mathbf{u}=\frac{\pi}{10}\left[\begin{array}{ll}1 & 1\end{array}\right]^{T}$ and initial conditions $\quad \mathbf{x}(0)=\left[\begin{array}{ll}0 & 0\end{array}\right]^{T}, \quad \mathbf{x}(0)=\left[\begin{array}{ll}1 & 0\end{array}\right]^{T} \quad$ and $\mathbf{x}(0)=\left[\begin{array}{ll}0 & 2\end{array}\right]^{T}$, respectively. It can be seen from the figures that the SDM exhibit irregular chaotic patterns on the phase plane. Compared to the fixed point case shown in Figure 1, the value or $r$ is the same. Figures $3 \mathrm{~g}$-3i show the state trajectories of a bandpass SDM with filter parameters $r=0.99$ and $\theta=0.0001$, input step size $\mathbf{u}=0.4\left[\begin{array}{ll}1 & 1\end{array}\right]^{T}$ and initial conditions $\mathbf{x}(0)=\left[\begin{array}{ll}0 & 0\end{array}\right]^{T}, \mathbf{x}(0)=\left[\begin{array}{ll}1 & 0\end{array}\right]^{T}$ and $\mathbf{x}(0)=\left[\begin{array}{ll}0 & 1\end{array}\right]^{T}$, respectively. It can be seen from the figures that the SDM also exhibit irregular chaotic patterns on the phase plane. Compared to the limit cycle case shown in Figure 2, the value or $r$ is the same. Hence, even though the value of $r$ is close to the unit cycle, different behaviors may occur.

Figure 4 show the spectra of the corresponding output sequences of the above examples. It can be seen from the 
figures that there are no periodic impulses on the spectra, which illustrates that these SDMs do not exhibit limit cycle behavior and do not suffer from audio tonal effects.

Although there are some spikes in some of spectra of output sequences of the above examples, by grouping those $\mathrm{AC}$ frequencies that producing spikes together to form a set, say $\wp$, and defining the tonal suppressing ratio as follows:

$$
T S R \equiv 10 \log _{10}\left(\frac{\int_{\omega \in[-\pi, \pi] \wp}|S(\omega)|^{2} d \omega}{\int_{\omega \in \wp}|S(\omega)|^{2} d \omega}\right),
$$

it can be checked easily that the tonal suppression ratios of the above SDMs are $15.9137 \mathrm{~dB}, 19.2737 \mathrm{~dB}, 17.7570 \mathrm{~dB}$, $6.8905 \mathrm{~dB}, \quad 6.8985 \mathrm{~dB}, 6.5896 \mathrm{~dB}, 7.7505 \mathrm{~dB}, 7.7816 \mathrm{~dB}$ and $7.7498 \mathrm{~dB}$, respectively

Since all the simulations are carried out using MATLAB under a 64 bit computer. The numerical rounding error is insignificant compared to the distance between the poles of the system matrices and the unit circle. For example, the numerical error due to a 64 bit computer is $2^{-64}$, while the distance between the poles of the system matrix and the unit circle is $10^{-4}$ for $r=0.9999$, the ratio is just $5.42 \times 10^{-16}$. Hence, the effect of numerical rounding error can be regarded as insignificant.

One possible implication of the results obtained in this paper is that it is not necessary to place unstable poles in the system matrices of bandpass SDMs to generate signals with rich frequency spectra in order to suppress unwanted tones from quantizers. It is shown in this paper that fractal or irregular chaotic signals can be generated via system matrices with strictly stable poles. Since the output sequences are aperiodic, which consist of rich frequency spectra, the unwanted tones could be suppressed using these aperiodic signals without the tradeoff of the stability of the systems.

\section{DISCUSSION AND CONCLUSION}

In this paper, we found that fractal or irregular chaotic patterns may be exhibited in the phase portrait even though the system matrices of bandpass SDMs are strictly stable. One implication of the results obtained in this paper is that we can generate signals with rich frequency spectra by using strictly stable system matrices and hence unwanted tones generated by the quantizers are suppressed. Thus limit cycles may be avoided without a tradeoff in the stability of the bandpass SDM.

\section{REFERENCES}

[1] Juan Carlos de Mateo García, Ana García Armada, "Effects of bandpass sigma-delta modulation on OFDM signals," IEEE Transactions on Consumer Electronics, vol. 45, no. 2, pp. 318-326, 1999.

[2] Roberto Maurino and Peter Mole, "A 200-MHz IF 11-bit fourth-order bandpass $\Sigma \triangle \mathrm{ADC}$ in SiGe," IEEE Journal of Solid-State Circuits, vol. 35, no. 7, pp. 959-967, 2000.

[3] Paolo Cusinato, Fabrizio Stefani and Andrea Baschirotto, "Reducing the power consumption in high-speed $\Sigma \Delta$ bandpass modulators," IEEE Transactions on Circuits and Systems-II: Analog and Digital Signal Processing, vol. 48, no. 10, pp. 952-960, 2001.
[4] Paolo Cusinato, Davide Tonietto, Fabrizio Stefani and Andrea Baschirotto, "A 3.3-V CMOS 10.7-MHz sixth-order bandpass $\Sigma \Delta$ modulator with 74-dB dynamic range," IEEE Journal of Solid-State Circuits, vol. 36, no. 4, pp. 629-638, 2001.

[5] Weinan Gao and W. Martin Snelgrove, "A 950-MHz IF second-order integrated LC bandpass delta-sigma modulator," IEEE Journal of SolidState Circuits, vol. 33, no. 5, pp. 723-732, 1998.

[6] Stephen I. Mann and Desmond P. Taylor, "Limit cycle behavior in the double-loop bandpass $\Sigma-\Delta$ A/D converter," IEEE Transactions on Circuits and Systems-II: Analog and Digital Signal Processing, vol. 46, no. 8, pp. 1086-1089, 1999.

[7] Orla Feely, "A tutorial introduction to non-linear dynamics and chaos and their application to sigma-delta modulators," International Journal of Circuit Theory and Applications, vol. 25, pp. 347-367, 1997.

[8] Orla Feely and Leon O. Chua, "The effect of integrator leak in $\Sigma-\Delta$ modulation," IEEE Transactions on Circuits and Systems, vol. 38, no. 11, pp. 1293-1305, 1991.

[9] Sǿren Hein, "Exploiting chaos to suppress spurious tones in general double-loop $\Sigma \Delta$ modulators," IEEE Transactions on Circuits and Systems - II: Analog and Digital Signal Processing, vol. 40, no. 10, pp. 651-659, 1993.

[10] Lars Risbo, "On the design of tone-free $\Sigma \Delta$ modulators," IEEE Transactions on Circuits and Systems-II: Analog and Digital Signal Processing, vol. 42, no. 1, pp. 52-55, 1995.

[11] Mariam Motamed, Seth Sanders and Avideh Zakhor, "The double loop sigma delta modulator with unstable filter dynamics: stability analysis and tone behavior," IEEE Transactions on Circuits and Systems-II: Analog and Digital Signal Processing, vol. 43, no. 8, pp. 549-559, 1996.

[12] Orla Feely, "Theory of lowpass and bandpass sigma-delta modulation," IEE Colloquium on Oversampling and Sigma-Delta Strategies for DSP, pp. 7/1-7/8, 23 November, 1995.

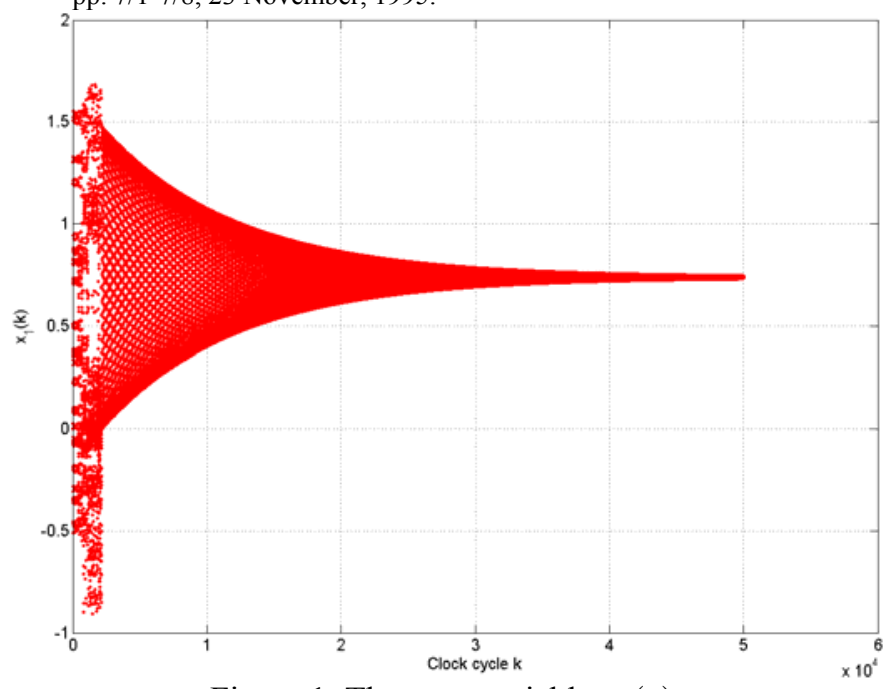

Figure 1. The state variable $x_{1}(k)$. 


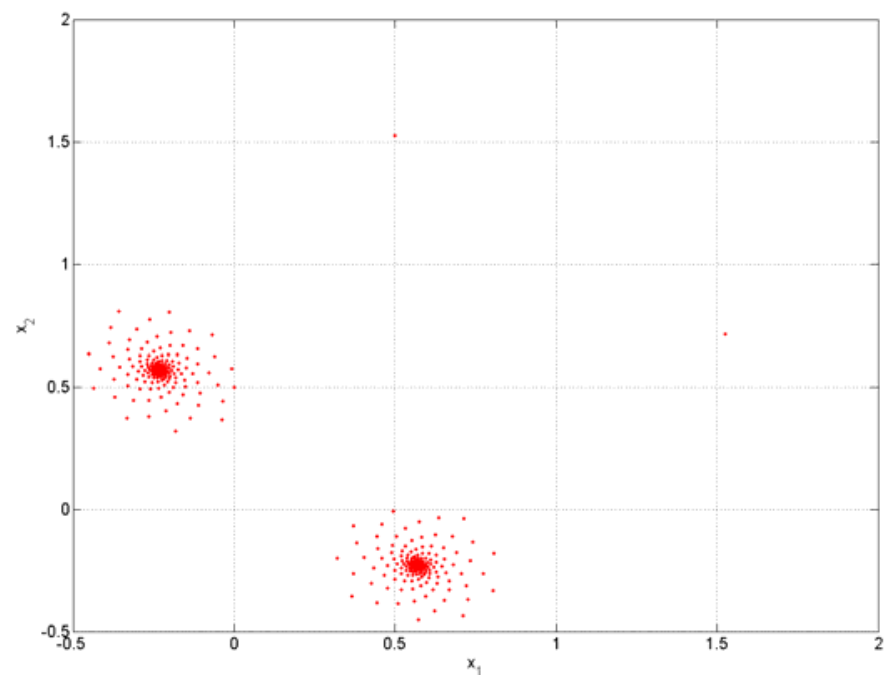

Figure 2. The phase portrait when $M=2$.
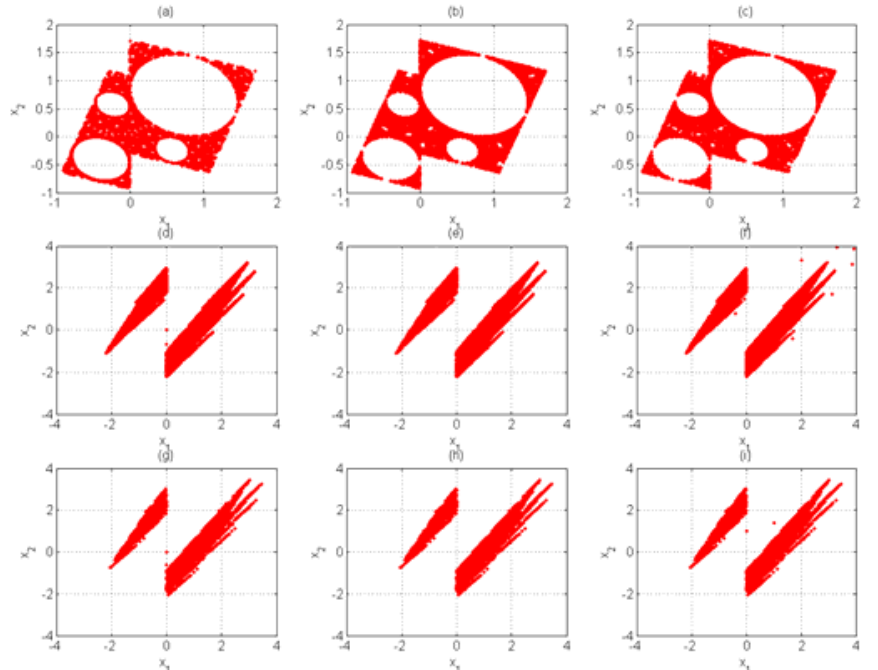

Figure 3. The phase portraits when output sequences are aperiodic.
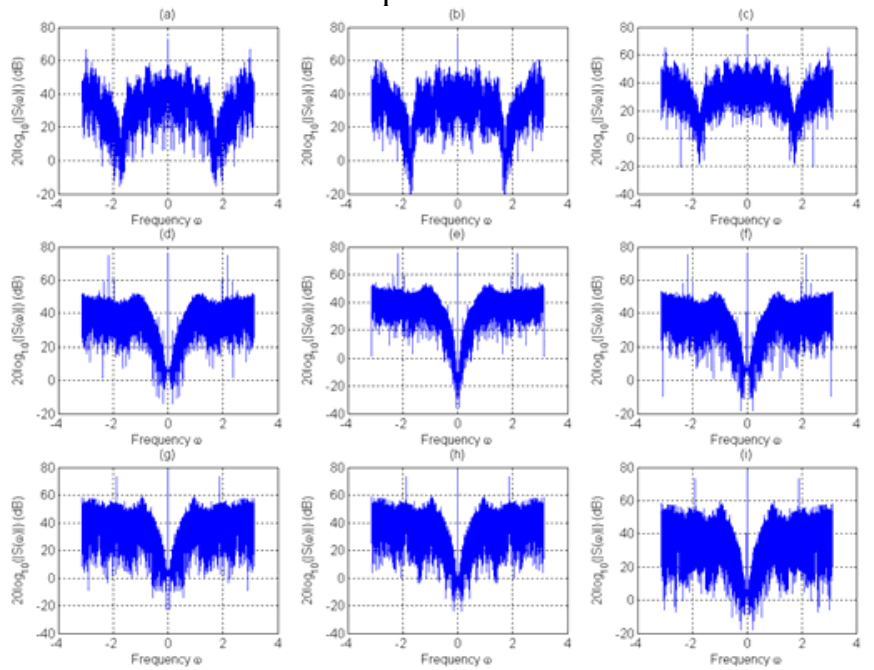

Figure 4 . The corresponding frequency spectra of the output sequences. 